\title{
Propuestas para vincular la ciudad y sus habitantes con lugares de arte contemporáneo
}

\author{
Proposals to link the city and its inhabitants \\ with the places of contemporary art
}

Resumen

Autora:

Laura Gallardo-Frías* lauragallardofrias@uchilefau.cl

*Universidad de Chile

Recibido: 01/Mar/2021 Aceptado: 10/Jun/2021
$\mathrm{L}$ os lugares destinados al arte contemporáneo: museos, centros de arte y centros culturales, entre otros, son lugares esenciales para nuestra cultura y tienen un desafío añadido por la propia definición de este tipo de arte, que lleva asociada una tensión con sus visitantes. El objetivo de este escrito es abrir la reflexión para vincular la ciudad y sus habitantes con los lugares del arte contemporáneo y mostrar algunas propuestas concretas. Para ello, se recoge una serie de reflexiones procedentes de una investigación multidisciplinar integrada por los ámbitos de: urbanismo, arquitectura, antropología e historia, que durante dos años analizó esta relación y propuso llevarla al taller de proyectos arquitectónicos de segundo año de la Universidad de Chile, con la finalidad de compartir hallazgos con los estudiantes y proponerles un ejercicio para realizar 'proyectos mediadores', es decir, propuestas arquitectónicas que vinculen los lugares de arte contemporáneo con la ciudad y sus habitantes.

Palabras clave: museos; centros de arte; centros culturales; proyecto urbano; proyecto arquitectónico.

\section{Abstract:}

The works of architecture that shelter art, reference is made to museums, art centers and cultural centers, among others, are essential places for our culture. Places destined for contemporary art have an added challenge by the very definition of this type of art, which is associated with a tension with its visitors. The objective of this writing is to open the reflection to link the city and its inhabitants with the places of contemporary art and show some concrete proposals. To do this, a series of reflections from a multidisciplinary research integrated by the areas of: urbanism, architecture, anthropology and history are collected, which for two years analysed this relationship and proposed taking it to the secondyear architectural projects workshop at the University of Chile, in order to share findings with students and propose an exercise to carry out 'mediating projects', that is, architectural proposals that link contemporary art places with the city and its inhabitants.

Keywords: museums; art centers; cultural centers; urban project; architectural project. 


\section{Introducción}

Los centros de arte, museos y centros culturales, entre otros tipos arquitectónicos que tienen programas enfocados al arte contemporáneo, si bien han sido y siguen siendo grandes motores urbanos, abriendo la posibilidad de desarrollo de nuevos sectores, también han generado en los últimos tiempos numerosas estrategias para continuar y fomentar su relación con su comunidad, el barrio y la ciudad, con la finalidad de atraer a los visitantes y llegar a constituirse como centros de encuentro social y cultural. Existen numerosos ejemplos internacionales sobre cómo estos lugares de arte contemporáneo han impulsado sectores de la ciudad (Almeida et al., 2012; Valenzuela et al., 2015; Ayala et al., 2020), en los cuales el vínculo con sus habitantes ha sido un factor clave para la regeneración de barrios y espacios públicos (Bueno, 2021; Hernández y De La Torre, 2021).

El objetivo de este escrito es abrir la reflexión sobre el vínculo de la ciudad y sus habitantes con los lugares del arte contemporáneo. Por una parte, desde una propuesta teórica, se exponen diferentes enfoques acerca de la significación y puesta en valor de estos tipos arquitectónicos, así como los resultados de una investigación conformada por un equipo de investigadores de los ámbitos de la arquitectura, urbanismo, historia y antropología, donde se dan a conocer factores relevantes para tener en cuenta en cada una de estas dimensiones. Por otra parte, se muestran algunos de los resultados obtenidos al llevar esta investigación a los estudiantes del taller de proyectos arquitectónicos, donde se realizaron propuestas concretas a partir de 'proyectos mediadores' que aspiran a vincular los museos, centros de arte y centros culturales con su barrio y sus habitantes.

\section{Marco Teórico}

Para comprender el significado de los lugares de arte contemporáneo es clave recordar que estos tipos arquitectónicos tienen su base en el museo. El término museo deriva de la palabra Musa (Corominas, 1987), remitiendo en su concepción inicial a "Mouseion, o templo de las Musas, término que originariamente significaba bosquecillo sagrado" (Rivière, 2009, p. 68). En función de las posibilidades que abren los museos hacia el vínculo con sus habitantes se puede establecer una clasificación en tres grandes grupos. El primero de ellos hace referencia a lugares vinculados con la quietud, con el interior, donde se encuentran las definiciones de: conservatorio, lugar de colección (Desvallées, 2000; Poulot, 2009), tesoro de los dioses y los hombres (Baldellou, 2014), salón, escuela (Valéry, 2005; Calaf y Gutiérrez, 2017), santuario (Hernández, 1992), cementerios y dormitorios públicos (Marinetti, 1909). El segundo grupo refiere a lugares abiertos al movimiento, la reflexión, donde se encuentran las comparaciones con: laboratorios (Collados, 2015), cámaras de maravillas (Ferrer, 2018), organismos extraordinarios (Montaner, 2003), lugar de participación, lugar de investigación, gabinetes de curiosidades (Weil, 1995), foros (Cameron, 1971), templos del placer (Rocco, 2018), casas de tesoros donde la verdad y la belleza se descubren, atmósferas donde se dan ideas estimulantes y el aprendizaje activo (Kisida et al., 2013; Dorrian, 2014), hacia la búsqueda de un sueño, una excitación (Newhouse, 1998). Y, por último, un tercer conjunto de definiciones ligadas a lugares de nuevas tendencias y programas, como: malls y supermercados culturales (Álvarez y Benjumea, 2011), objetos eróticos (Guasch, 2008), hasta refugios placenteros, catalizadores de nuevas ideas, moderadores y facilitadores (García, 2015), entre otros.

El Consejo Internacional de Museos (ICOM), desde su creación en 1946, ha revelado la relación de los museos con la sociedad como uno de sus elementos fundamentales (Mairesse y Desvallées, 2007; Botte et al., 2017). En la definición actual, contenida en los estatutos de esta institución, adoptados por la 22a Asamblea general de Viena,

un museo es una institución permanente, sin fines de lucro, al servicio de la sociedad y abierta al público, que adquiere, conserva, estudia, expone y difunde el patrimonio material e inmaterial de la humanidad y su ambiente con fines de estudio, educación y recreo (ICOM, 2007, p.5)

Las obras arquitectónicas destinadas a albergar arte son espacios que se constituyen en lugar a partir del vínculo que establecen con sus visitantes. Además de los habitantes, el vínculo con el barrio y la ciudad es fundamental.

Es así como la nueva museología cuestiona al museo como monumento cerrado y expande el campo de musealización a toda la ciudad. Es importante hacer un llamado de atención para evitar la generación áreas de gentrificación (Luque, 2018; Fiori, 2016) a partir de distintos espacios culturales.

Los lugares de arte son 'piezas motoras' para las ciudades (Layuno, 2003; Galland, 2014; Falcón, 2019; Gallardo et al., 2019), capaces de regenerar, transformar y dotar de identidad y vida a los barrios en los que se insertan. Sin embargo, los lugares de arte destinados al arte contemporáneo -se hace referencia a museos, centros de arte y centros culturales, entre otros tipos arquitectónicos-, tienen una dificultad particular para atraer a los visitantes, por la propia definición del arte contemporáneo, como indica Badiou:

El arte contemporáneo va a tomar, entonces, otra dirección, que estará ligada a los efectos que produce: el arte no será un espectáculo, ni una detención del tiempo, más bien será lo que compromete en el tiempo mismo y produce efectos en el tiempo. Se podría incluso decir que el arte clásico es una instrucción para el sujeto, una lección para el sujeto y, en cambio, la obra contemporánea apunta hacia una acción que cuestiona y transforma al sujeto (Badiou, 2013, p. 3).

Así, los lugares que cobijan arte contemporáneo pasan a ser aparatos (Déotte, 2012) más de preguntas que de respuestas (Rispal, 2009), tensionando el vínculo con sus visitantes (Villarreal, 2007) y, por tanto, con su entorno. 
El vínculo de los lugares de arte contemporáneo con los visitantes y la ciudad fue estudiado durante dos $a_{\text {ños }}{ }^{1}$ por un equipo multidisciplinar que investigó cinco obras emblemáticas de la ciudad de Santiago de Chile. El objetivo fue analizar, desde la perspectiva del lugar, a partir de la dimensión histórica, urbana, arquitectónica y sociocultural, obras emblemáticas de arquitectura pública destinadas al arte contemporáneo en la vinculación con sus visitantes y su contexto. De esta investigación se obtuvieron factores relevantes a considerar para mejorar el vínculo de los lugares de arte con sus visitantes (Gallardo, 2020). En la dimensión histórica se destacan tres factores a tener presentes: la historia del terreno, la historia de la edificación y la historia de la institución. Para la dimensión urbana, será importante tener en cuenta: el clima, la topografía, el emplazamiento, la legibilidad, la relación entre llenos-vacíos y alturas, los usos de suelo, el sistema de red de equipamientos de arte, la conectividad vial y de transporte, los flujos de personas, las áreas verdes y los factores sensoriales como: vistas, colores, sonidos, olores, texturas, entre otros. En lo que respecta a la dimensión arquitectónica los factores relevantes son: la geometría, los accesos, las fachadas, el programa, los espacios servidores y servidos, la estructura, los materiales, los colores, las texturas, la luz natural y artificial, las vistas, la acústica y la climatización. Y en la dimensión sociocultural, que se destacó como una dimensión central, pues entrecruza al resto de dimensiones, será clave tener presente: los tipos y usos del espacio, los estilos y tiempo de visita, el mapa de actores y estructura de relaciones, los conflictos y solidaridades, las relaciones entre visitantes y objetos de arte, las normas de permanencia y pautas de comportamiento, los tipos de trayectorias de recorrido, los usos de tecnología de registro, valoraciones, y recomendaciones para el lugar del arte.

Se decidió llevar esta investigación al taller de proyectos arquitectónicos de la Universidad de Chile, para compartir con los estudiantes los hallazgos y formular un ejercicio donde ellos pudieran realizar propuestas arquitectónicas con la finalidad de vincular lugares de arte contemporáneo con sus habitantes y su entorno.

\section{Métodos}

En una primera fase se presentó a los estudiantes e marco teórico con la revisión de la significación de los lugares del arte y su importancia para las ciudades y sus habitantes. Los investigadores fueron al taller, durante el primer semestre del 2018, para poner de manifiesto las diferentes dimensiones involucradas en esta investigación. Las historiadoras resaltaron la importancia de los hechos vinculados a los distintos lugares (Özkan y Akalin, 2019) para el arte contemporáneo, su huella pasada y su significación en el presente que permitía proyecciones futuras. Los arquitectos subrayaron la relevancia de las dimensiones urbana y arquitectónica para comprender estos tipos arquitectónicos en estrecha relación con su tejido urbano y la ciudad. Los antropólogos dieron cuenta del componente fundamental de los visitantes, ya que sin ellos los lugares de arte morirían.

\footnotetext{
${ }^{4}$ Proyecto de investigación Fondecyt №: 11170140, titulado: “El lugar del arte contemporáneo en Santiago de Chile". Análisis de obras emblemáticas de arquitectura pública y su vinculación con los habitantes desde la dimensión histórica, arquitectónica, urbana y socio-cultural".
}

Así, con todos los fundamentos teóricos, se les propuso a los estudiantes analizar diferentes lugares emblemáticos que cobijan arte contemporáneo en Santiago de Chile. Para este artículo, con la finalidad de revisar tres tipos distintos, se han seleccionado: Museo de Arte Contemporáneo de Quinta Normal (MACQN), Centro Cultural la Moneda (CCLM) y Centro de Arte Contemporáneo de Cerrillos (CNACC). Para iniciar el estudio de estos centros se propuso a los estudiantes una pauta de análisis basada en siete puntos (Gallardo, 2014): Genius loci, donde se revisan los hechos relevantes de la historia para comprender el espíritu del sector a analizar; Movimiento - quietud, se estudia cómo el lugar de arte se vincula a la ciudad y al barrio, las principales arterias y formas de conexión, y también los principales puntos de reposo del sector; Análisis sensorial, con la finalidad de entender el recorrido del sol, los colores, texturas, olores, y vistas principales, entre otros; Elementos construidos, a fin de comprender el tejido urbano donde se inserta el caso de estudio, los tipos de suelo, relación de sectores públicos y privados, llenos y vacíos; Zonas verdes, con las distintas especies, formas y alturas; Estudio etnográfico, donde los estudiantes, guiados por los antropólogos, fueron a conversar con los habitantes del sector para conocer las distintas perspectivas, necesidades y comprender mejor el sector de análisis, para lo que realizaron entrevistas etnográficas; y el último punto corresponde a una síntesis de los principales hallazgos encontrados que den pistas para realizar la propuesta arquitectónica. Cada uno de estos puntos de análisis fue sintetizado en una lámina, conformando para cada sector una documentación de siete láminas en total.

Este análisis permitió conocer y comprender cada uno de los lugares de arte, para, en la siguiente fase de proyectación, llegar a generar 'proyectos mediadores', es decir, propuestas arquitectónicas que acercaran o vincularan los distintos casos de estudio a la ciudad y sus habitantes.

\section{Resultados}

Para el trabajo de análisis de cada lugar de arte se trabajó en equipo, conformado por tres estudiantes, y después cada uno de ellos realizó individualmente un proyecto mediador para el centro que había analizado. Se muestra a continuación el análisis de cada centro de arte, seguido por dos proyectos mediadores.

Se comienza por el Centro Nacional de Arte Contemporáneo de Cerrillos (CNACC). Los estudiantes relevaron en su ubicación la búsqueda de rescatar su historia al instalarse como el primer aeropuerto de Chile, así como una posibilidad de descentralizar el arte en de la región metropolitana al situarse en la comuna de Cerrillos, cercano al metro, junto al museo Aeronáutico y el edificio de las Fuerzas Aéreas de Chile. Ponen de relevancia su ubicación frente al gran parque bicentenario de cerrillos y destacan la diferente percepción de este Centro desde el oriente y el poniente: la primera más relajada, libre y natural desde el parque, en contraste con el poniente donde se percibe de manera más cerrada, confusa y poco atractiva. El estudio etnográfico, realizado a personas que transitan por el barrio, indicó que casi un $70 \%$ no conoce todavía el Centro. Entre las principales características de 
los entrevistados, los estudiantes relevaron que el CNACC adolece de una buena difusión dentro de la ciudad. Entre las fortalezas destacan que el centro se ubica en una comuna en desarrollo, con una historia relevante y grandes espacios sin uso. Como oportunidades advierten la posibilidad de proyectar nuevos espacios de recreación, comercio y esparcimiento para atraer a mayor número de visitantes. Finalmente presentan como objetivo principal para este Lugar de arte el potenciar la vista, tanto al Centro como a la comuna, fortaleciendo la idea de descentralizar el arte y la cultura. Para concretizar este objetivo, las propuestas de partida se basan en la creación de hitos representativos del lugar para servir de referencia, y el desarrollo de una red de museos que permita generar un gran foco cultural urbano (Revisar Figura 1 donde se detallan los 7 puntos analizados).
Proyecto 1 CNACC: "Paseo de la luz". La estudiante subraya que uno de los principales problemas de este Centro radica en que el acceso pasa desapercibido por la valla ubicada en el acceso principal situado en la Av. Pedro Aguirre Cerda, la cual impide la percepción a los transeúntes del propio Centro y de su entrada poniente. Así, plantea un proyecto cuya finalidad es prolongar la pérgola existente hacia la calle como dos brazos que salen a buscar y a abrazar a los transeúntes para invitarlos a entrar. Estos brazos se trazan como elementos que acogen y ofrecen un recorrido con sombras, diseñadas a través de vidrios de colores y espejos que producen interesantes efectos lumínicos, para conducir a los visitantes hasta el centro (Figura 2).

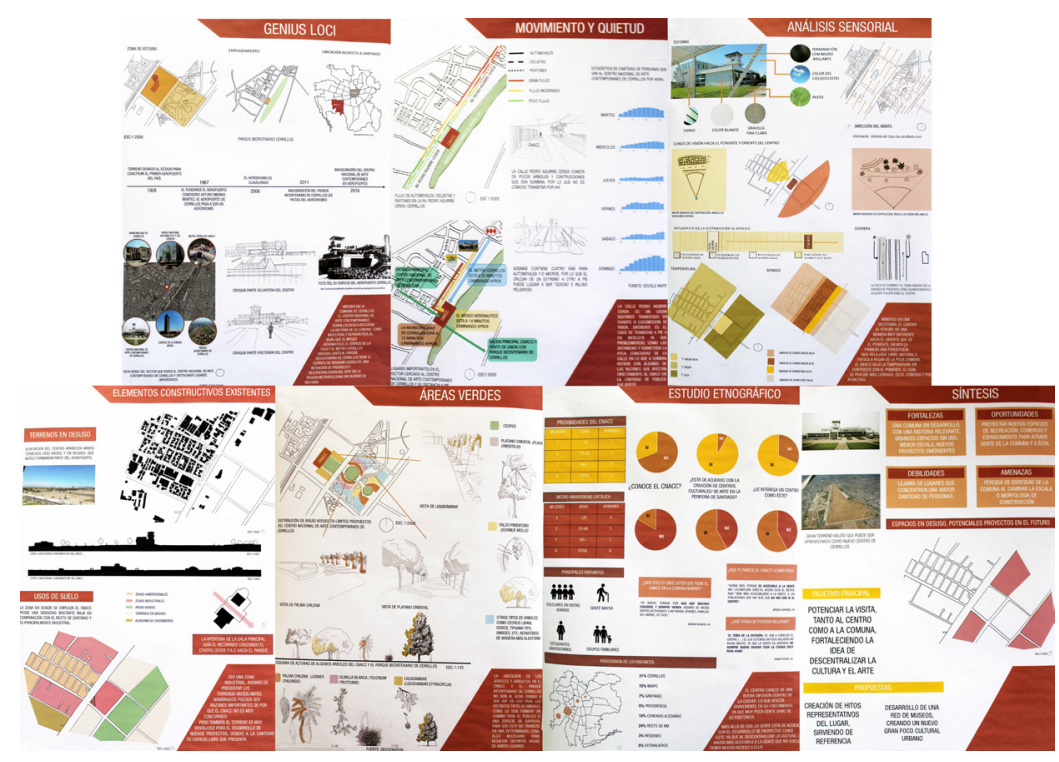

Figura 1: Análisis del Centro Nacional de Arte Contemporáneo de Cerrillos (CNACC) a partir de 7 puntos principales. Estudiantes taller 3

Fuente: Elaboración propia

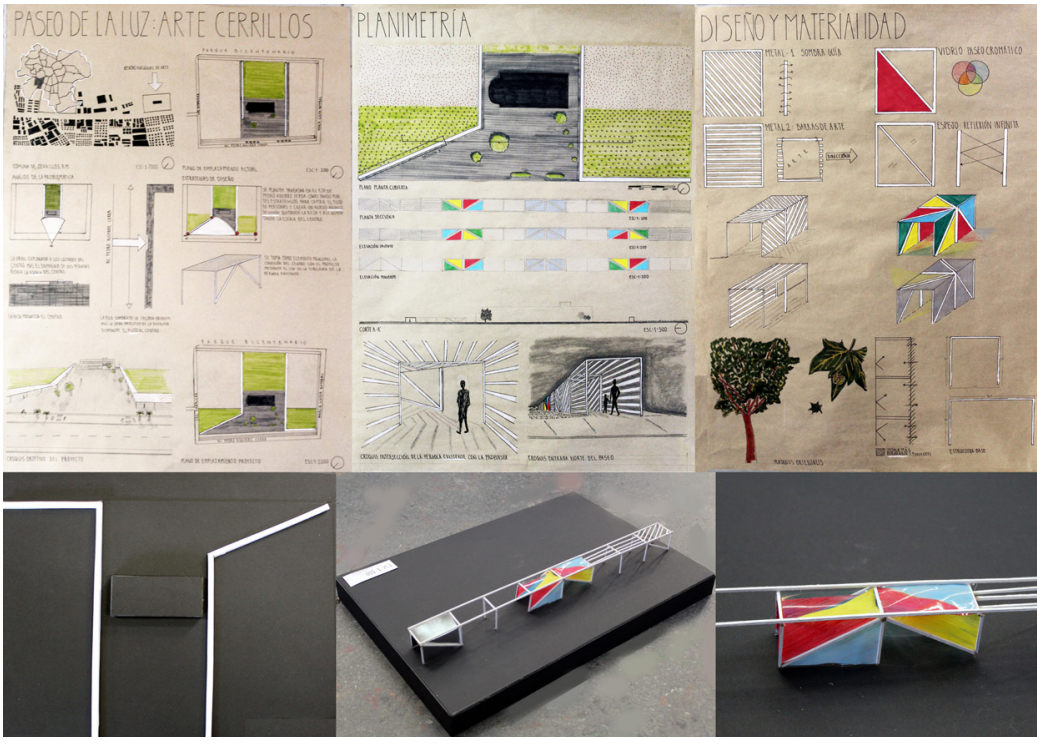

Figura 2. Proyecto 1 CNACC: "Paseo de la luz". Estudiante Javiera Morales

Fuente: Elaboración propia 
Proyecto 2 CNACC: "Promenade del arte". Este estudiante llega a la conclusión que la vereda de la Av. Pedro Aguirre Cerda, en particular el tramo que va desde el metro hasta el Centro, si bien tiene un gran potencial por su ancho y por los elementos de mobiliario público existentes, es demasiado árida pues no hay árboles y no se anuncia ni se promueve el CNACC. Así, propone sacar el arte a la calle para mostrar a los caminantes distintas obras, generando sombras, y también para invitarlos a entrar a seguir descubriendo otras obras al interior del Centro (Figura 3).

Museo de Arte Contemporáneo de Quinta Normal (MACQN). Los estudiantes destacan en su análisis que se emplaza dentro del parque Quinta Normal, donde confluyen tres grandes comunas y existe una red de museos, además de ser un sector patrimonial y turístico. Destacan un alto flujo vehicular y peatonal debido a su cercanía con la Av. Matucana, el metro, el hospital y colegios. También existen espacios de permanencia siendo los más relevantes los bancos del parque. El tejido urbano donde se inserta este Museo destaca por su densidad y su proximidad con el parque de la Quinta Normal, donde existen numerosas y diferentes especies vegetales. Los estudiantes advierten que un $60 \%$ de las 20 personas entrevistadas no conoce el MAC, es el menos frecuentado de los museos del sector, y proponen que puede ser debido a que este Museo no cuenta con un acceso desde el parque, se encuentra retranqueado de la av. Maturana rodeado con una reja que lo aleja de la vereda, y además se aprecia un estado de deterioro. Sin embargo, los estudiantes son capaces de rescatar su potencial como edificio histórico y contendor de una valiosa colección nacional, así como de la posibilidad de revalorizar su entono cercano (Revisar Figura 4 donde se analiza el centro en su entorno)

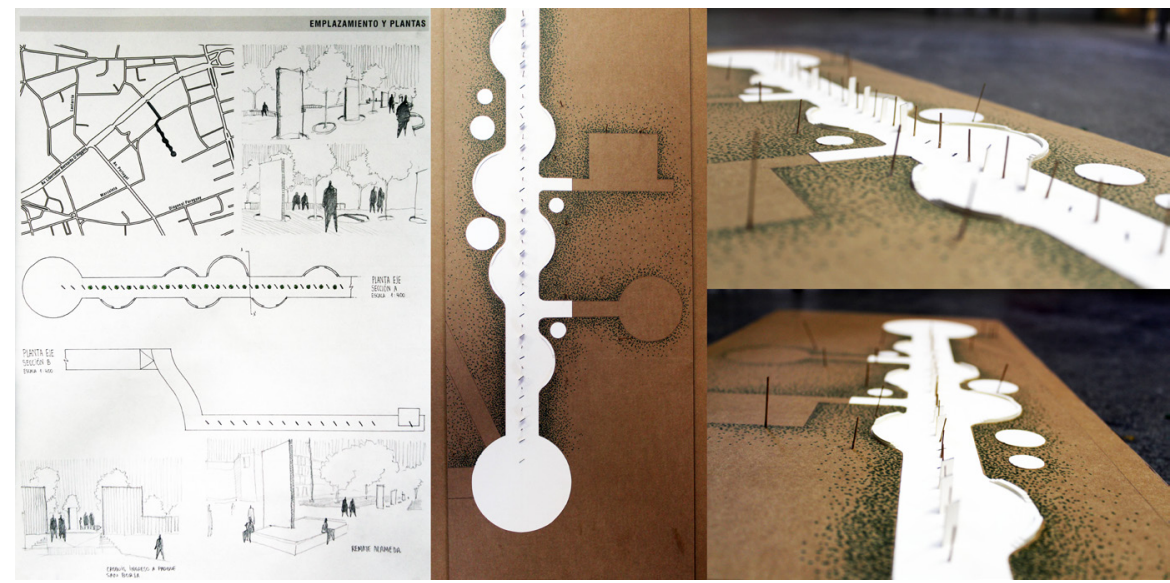

Figura 3. Proyecto 2 CNACC: "Promenade del arte". Estudiante Felipe Vásquez Fuente: Elaboración propia

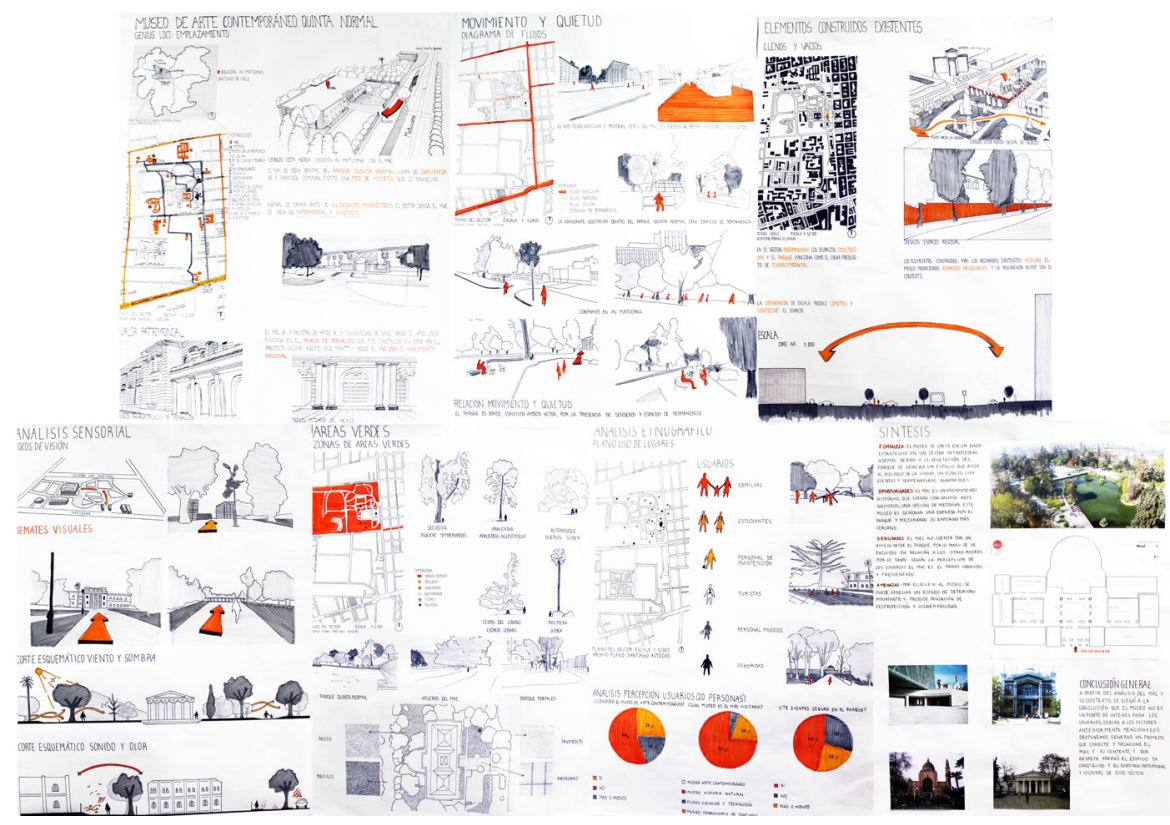

Figura 4. Análisis del Museo de Arte Contemporáneo de Quinta Normal (MACQN) a partir de 7 puntos principales. Estudiantes taller 3

Fuente: Elaboración propia 
Proyecto 1 para el MACQN: "Pabellón Contemporáneo". La estudiante llega a la conclusión que la reja aísla al museo de su entorno y propone trabajar el límite del Museo con la finalidad de trazar nuevos caminos y potenciar un foco visual hacia el museo, reemplazando y transformando la reja existente por un pabellón. El proyecto del pabellón se organiza en tres sectores o tipologías: la primera de permanencia, destinada al uso del arte urbano como e grafiti, el baile, entre otras; el segundo sector se organiza como una zona de transición dedicada a diferentes muestras centrales de arte contemporáneo; y el tercer sector se concibe como una trama compacta, dedicado a muestras aéreas. Se destaca de este proyecto cómo la trama que conforma el pabellón se va graduando, en conjunto con el pavimento que la acompaña, para dar énfasis al arte contemporáneo en su acercamiento a los transeúntes y abriendo el acceso del MACQN a los visitantes y al entorno (Figura 5).
Proyecto 2 MACQN: "La permanencia del arte". Esta estudiante subraya como principal debilidad la desconexión del Museo con el parque donde se ubica, y ve como oportunidades el gran flujo de personas que transita por el sector, el parque con sus variadas especies de árboles y la posibilidad de generar espacios de permanencia en el mismo. Propone como estrategias de diseño: sacar la reja existente que rodea al Museo para acercarlo a su entorno, generar nuevos senderos para la conexión del MAC con el parque, y la creación de un elemento que acerque el arte a las personas y que potencie la permanencia. Todo esto lo concretiza en un proyecto conformado por unos bancos situados estratégicamente en senderos que conectan e Museo con el sector. Estos bancos permiten sentarse a contemplar el parque, el entorno y las obras de arte colocadas en su parte central (Figura 6).

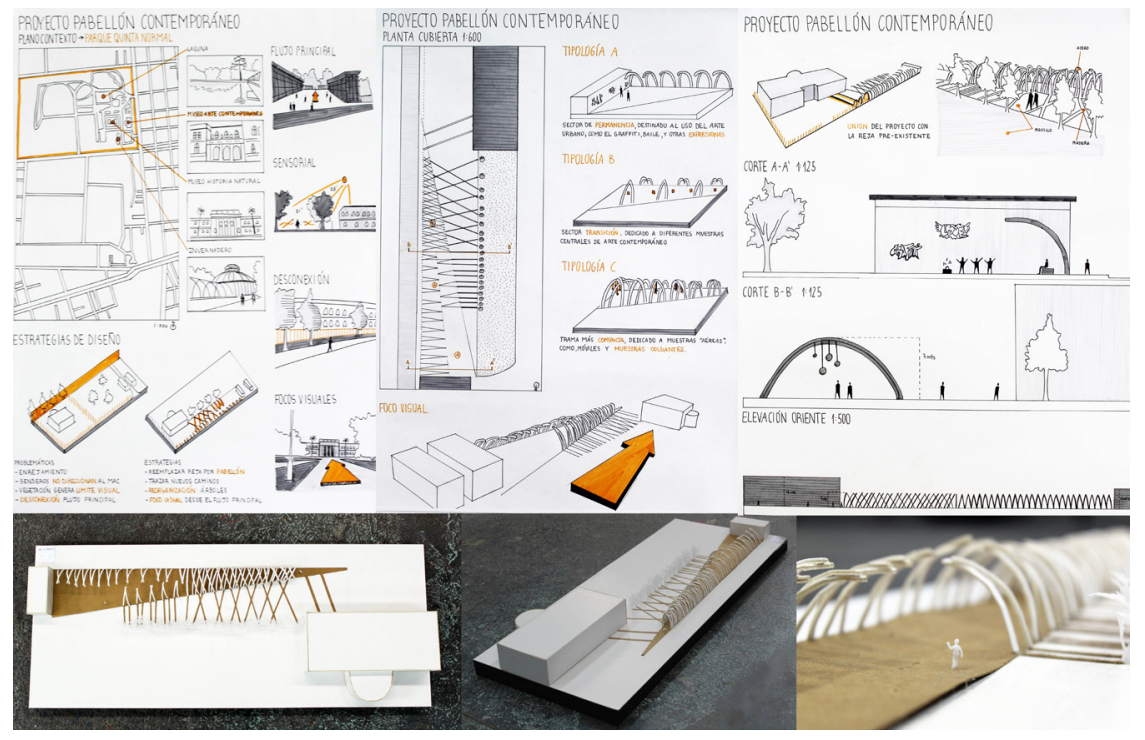

Figura 5. Proyecto 1 MACQN: "Pabellón Contemporáneo". Estudiante Fernanda Painemi Fuente: Elaboración propia

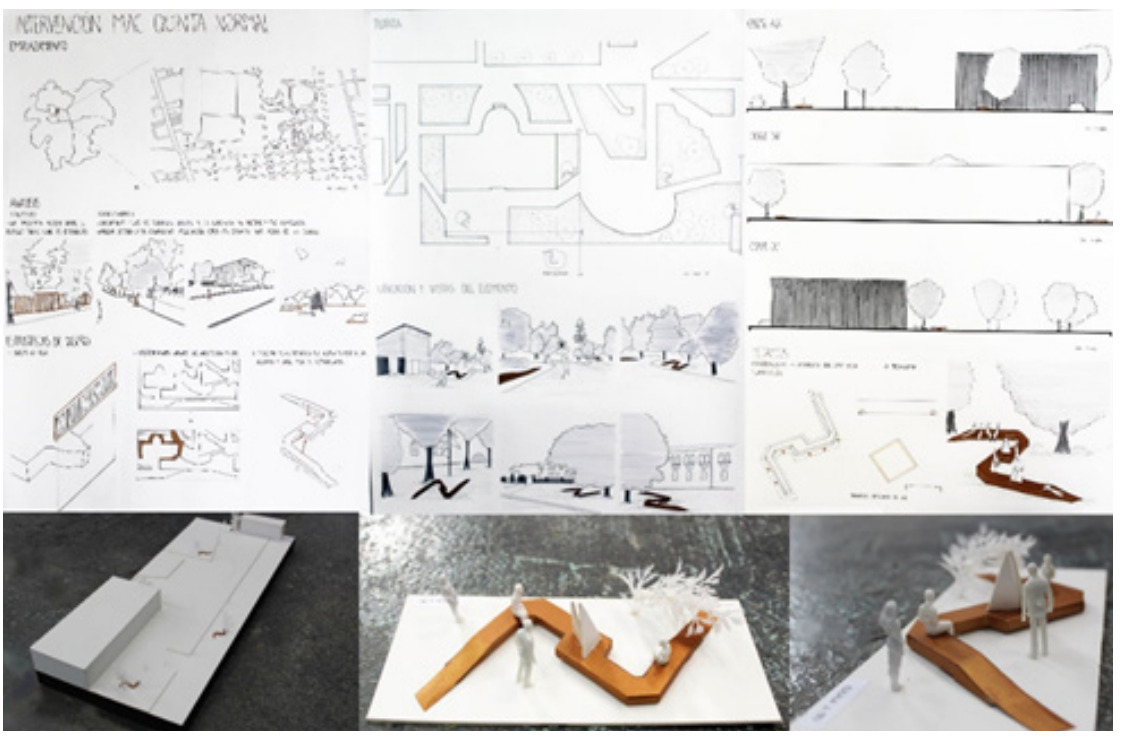

Figura 6. Proyecto 2 MACQN: "La permanencia del arte". Estudiante Paola González

Fuente: Elaboración propia 
Centro Cultural La Moneda (CCLM). En el análisis realizado los estudiantes rescatan la importancia de la ubicación de este Centro, ya que se encuentra en la principal arteria del país, La Alameda, y además se vincula con el Palacio de Gobierno de Chile. Destacan la conformación de este espacio por los edificios aledaños que lo convierten en un gran vacío, que también se genera al interior de este Centro. Existe un gran flujo vehicular que produce altos niveles de ruido, el cual va disminuyendo al acceder al CCLM a través de las rampas que se sumergen en la tierra y la cascada de agua que amortiza todos los sonidos exteriores. En el estudio etnográfico los estudiantes resaltaron el distinto tipo de transeúntes del sector; la mayoría de ellos lo conoce (80\%), y lo define como "lindo y seguro", sin embargo, también destacan la dificultad de encontrar el acceso, característica subterránea que señalan como una de sus principales debilidades para la conexión con su entorno. No obstante, su ubicación, la trama urbana y el acceso gradual, se convierten en grandes posibilidades a explorar (Revisar Figura 7 donde se analiza el CCLM en su entorno).

Proyecto 1 CCLM: "Vivir el arte". Para esta estudiante la principal debilidad de este Centro es la dificultad de advertir el acceso desde la calle y también que pase desapercibido el hecho de que, además de ser un centro cultural, tiene exhibiciones de gran interés. Así, propone una estructura mediante postes y tensores que permite conectar la calle con el acceso al CCLM y además sacar el arte a la calle utilizando esta estructura a modo de "colgar" el arte para hacer partícipes a los transeúntes e invitarlos a seguir descubriendo el arte al interior (Figura 8).

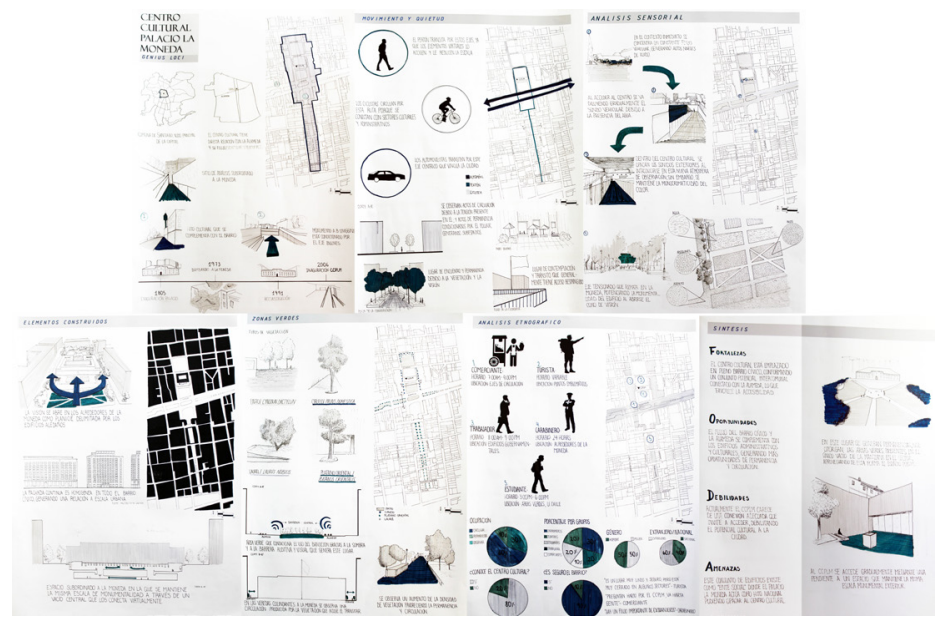

Figura 7. Análisis del Centro Cultural la Moneda (CCLM) a partir de 7 puntos principales. Estudiantes taller 3 Fuente: Elaboración propia

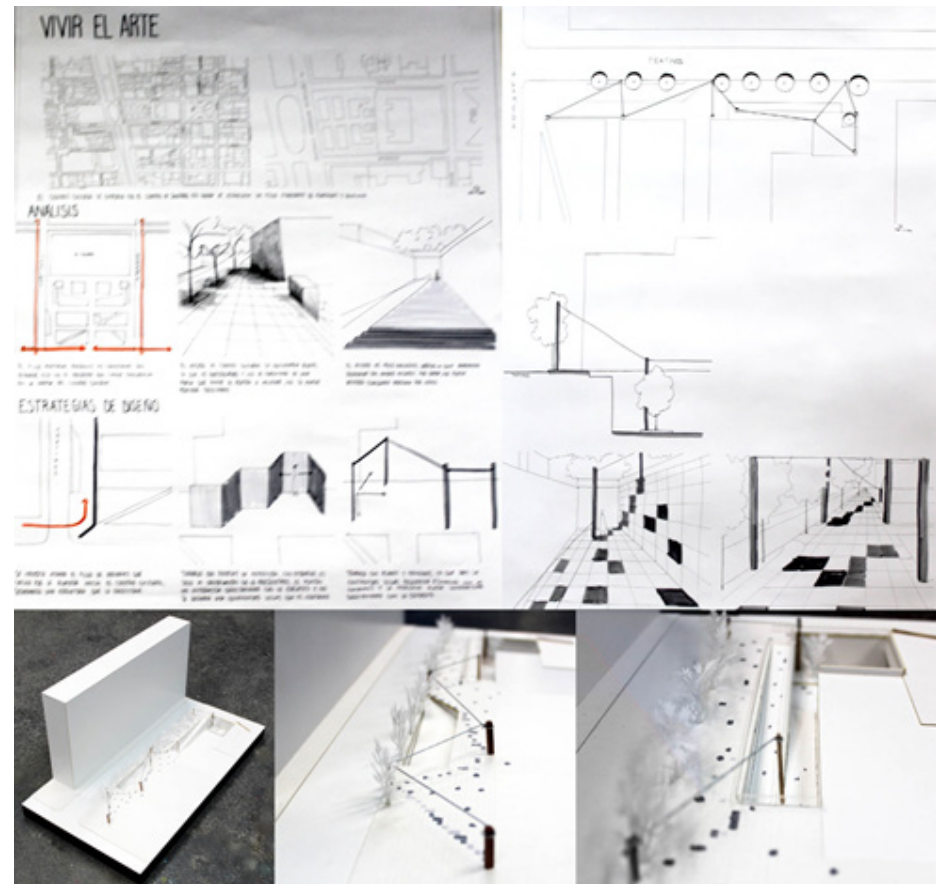

Figura 8. Proyecto 1 CCLM: "Vivir el arte". Estudiante Teresa Reyes Fuente: Elaboración propia 
Proyecto 2 CCLM: "Recorrido del arte". El estudiante hace partir su proyecto con una pregunta: ¿Cómo potenciar el acceso al museo de forma eficiente y vinculando el arte? Para responderla estudia los flujos peatonales del sector y propone un proyecto que consiste en crear una trama que una los puntos relacionados con el arte en todo el entorno. La propuesta consiste en una intervención en el suelo a partir de líneas directamente vinculadas con la densidad de los flujos. La técnica que propone es la misma que se utilizó en la intervención del proyecto paseo Bandera con el uso de grafiti "motanana 94", e incluye también iluminación nocturna que potencia la trama propuesta (Figura 9).

\section{Discusión}

Lo que se inició como un experimento, al llevar a un curso de pregrado una investigación en curso de un equipo profesional financiada (Fondecyt), se convirtió en una valiosa experiencia para todos. Se pone as en valor la importancia de la educación en artes y en estética desde distintas miradas (Huerta, 2018). El hecho de invitar a los investigadores al taller, sumado a visitar diferentes museos y centros de arte, motivó muchísimo a los estudiantes, quienes fueron capaces de hacer cosas increíbles, ya que además de este ejercicio realizaron un estudio de referentes, a partir de láminas y maquetas de museos emblemáticos de todo el mundo, que acabó siendo expuesto en el Museo de Arte Contemporáneo del Parque Forestal, en el Centro Nacional de Arte Contemporáneo de Cerrillos y en el Hall de la Facultad de Ciencias Físicas y Matemáticas de la Universidad de Chile. Con esto se pone en valor, por una parte, lo interesante que puede ser llevar a otro ámbito una investigación, por la gran retroalimentación que se genera para todos y, por otra parte, se destaca el gran potencial que tienen los estudiantes, pues fueron capaces de observar, analizar, comprender y sintetizar puntos relevantes para vincular los lugares de arte con sus visitantes y entorno, con la finalidad de proponer 'proyectos mediadores'.
Los estudiantes comprendieron la importancia que tiene el 'invitar a entrar' para un lugar de arte contemporáneo. Así, se observa que en sus proyectos se repiten propuestas de ampliar, remarcar o mostrar el acceso de mejor forma para acercarlo, y con él el centro completo, hacia sus habitantes y su barrio; propuestas que fueron concretizadas a través de asomar brazos, generar un paseo-pabellón asociado, marcar el pavimento o generar elementos de mobiliario público para admirar obras de arte también desde la quietud. Otra propuesta que se repitió en el taller fue la de sacar el arte a la calle, acción que se echa de menos en las inmediaciones de la mayoría de los lugares de arte de Santiago de Chile.

De este modo se subraya como uno de los hallazgos encontrados en este campo de estudio la importancia de los accesos para los lugares de arte contemporáneos; que sean legibles, es decir, que se puedan identificar con claridad tanto desde lejos como desde las inmediaciones, así como la señalética asociada al centro que, en conjunto con sus elementos arquitectónicos (puertas, transparencia, porosidad, marquesinas, elementos que guíen hacia el acceso, entre otros), lleguen a invitar a entrar, abriéndose hacia los habitantes, el barrio y la ciudad.

Al iniciar el taller, la mayoría de los estudiantes no conocía los lugares de arte que íbamos a estudiar, sobre todo el CNACC, inaugurado tan solo dos años antes. Pero ellos mismos fueron capaces de ir valorando el potencial de los centros estudiados para que todas las personas los pudieran visitar, ya que una vez que los conocieron se dieron cuenta de que se les 'abría un mundo', tal como ellos mismos relataron. Así, los estudiantes, comprendieron que los lugares de arte constituyen hitos para las ciudades, y pusieron de relevancia, mediante sus propuestas mediadoras, la vinculación con su barrio y habitantes.

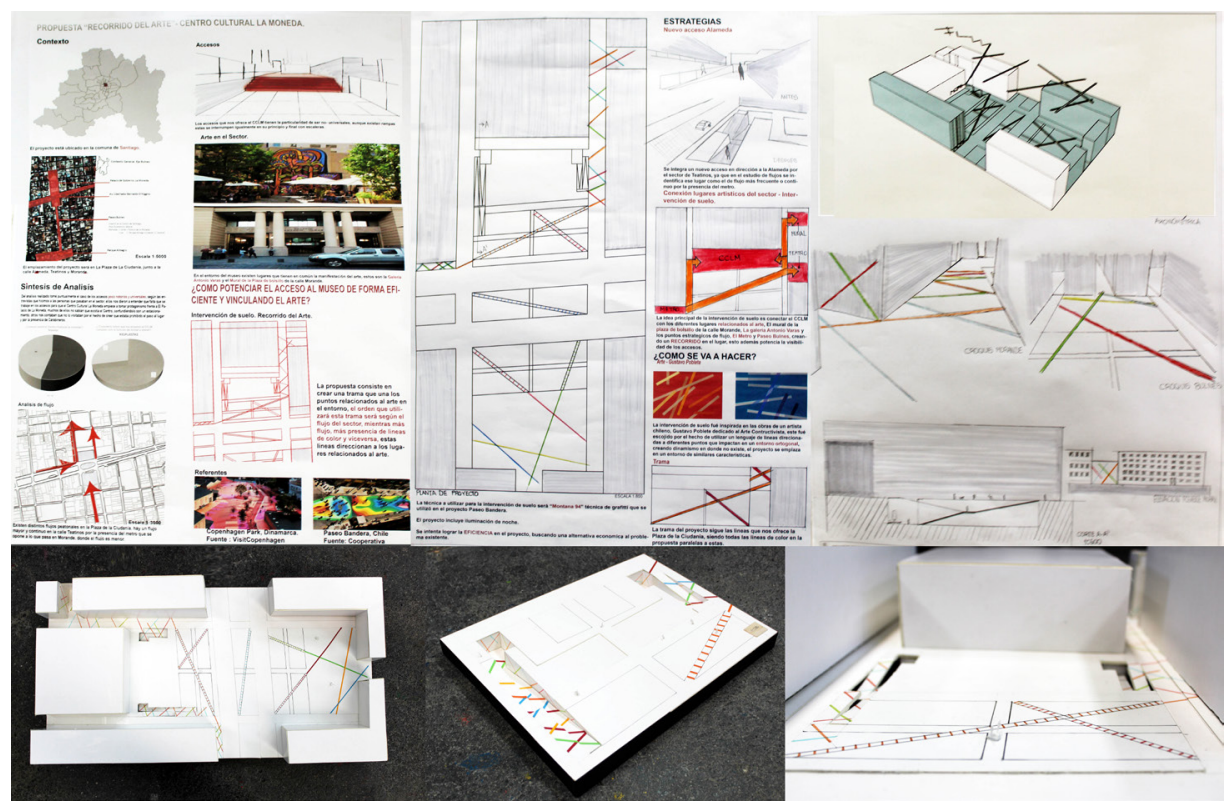

Figura 9. Proyecto 2 CCLM: "Recorrido del arte". Estudiante Alexis Lagos

Fuente: Elaboración propia 


\section{Conclusiones}

Las obras arquitectónicas destinadas al arte, además de ser contenedores de tesoros, se abren a la posibilidad de cobijar a sus visitantes, operando como receptáculos de sentido, es decir, como auténticos lugares.

Los lugares destinados al arte contemporáneo poseen además la particularidad de suscitar una reflexión en sus visitantes. Hay que tener presente la riqueza de estos tipos arquitectónicos, donde se vinculan entre sí distintas dimensiones, entre las que destacan la dimensión histórica, la institucional, la dimensión urbana, la arquitectónica y la dimensión sociocultural

Con la pandemia actual los lugares del arte se cerraron. Sin embargo, gracias a distintas redes sociales e internet abrieron sus puertas más que nunca, haciendo posible visitar desde nuestras casas museos emblemáticos de todo el mundo, y mostrando el gran valor de estos tipos arquitectónicos para la sociedad (Unesco, 2020).

Se destaca la relevancia que poseen los lugares de arte para la ciudad y sus habitantes, pues conservan la memoria, permiten un continuo aprendizaje, fortalecen el vínculo entre pasado-presente-futuro, ya que están abiertos a nuevas interpretaciones y significados. Por ello es fundamental considerarlos piezas esenciales de las ciudades, pues constituyen auténticos crisoles donde se fusionan las distintas claves de la cultura.

En este pequeño escrito se da cuenta de las grandes posibilidades que existen, así como de la necesaria continua investigación, abierta a los requerimientos de cada momento, para mantener vivos los lugares de arte a través del vínculo de la ciudad y sus habitantes.

\section{Agradecimientos}

Este artículo surge a partir del proyecto de investigación financiado por el Fondo Nacional de Desarrollo Científico y Tecnológico de Chile, FONDECYT N.o 11170140, título: "El lugar del arte contemporáneo en Santiago de Chile. Análisis de obras Emblemáticas de arquitectura publica y su vinculación con los habitantes desde la dimensión histórica, arquitectónica, urbana y sociocultural".

Agradecimientos a todos nuestros estudiantes del taller 3 del 2018 (tercer semestre) de segundo año: Armijo Silva, Almendra Aline. Bustos Bustos, Nicolás Sebastián. Chen, Liqiong. Flores Fuentes, Arantza Almendra. Forno Collao, Martina. González Pérez, Paola Antonia. Lagos Orellana, Alexis Gabriel. Mardones Acevedo, Valentina Stefany. Mora Canales, Rachel Javiera. Morales Bravo, Javiera Andrea. Núñez Maldonado, Catalina Belén. Olivares Hermosilla, Silvana Camila. Palacios Sanhueza, Lucas Vicente. Reinoso Lagos, Isidora Francisca. Renis Barrientos, Dominique Andrea. Reyes Serrano, Teresa De Jesús. Rojas Cortez, Bárbara Sofía de Jesús. Ruiz Vásquez, Lorena Andrea. Santa María Izquierdo, Domingo José. Sepúlveda Nieto, Estefanía Isabel. Silva Vera, Jesús Ignacio. Tapia Pastrián, Natalia Andrea. Torres Álvarez, Milka Saray. Urrea Navarro, Valentina Daniela. Vallejos Mena, Oscar Ignacio. Vargas Zúñiga, Javier Hernán.
Vásquez Orellana, Felipe Ignacio. Zurita Aguilera, Carolina Jesús.

Equipo docente. Ayudante: Luís Pérez Huenupi. Monitores: Maximiliano Andrés Meléndez Martínez, Nelly Leiva Mardones y Paula Sagrista Hernández

Investigadores Fondecyt: María Isabel Toledo Jofré (Dra. Ciencias de la Educación), Consuelo Figueroa Garavagno (Dra.(c) Historia), Luís René Pérez Huenupi (Arquitecto), Javier Vera Bravo (Antropólogo), Paulina Román Manzo (Mg. Comunicación).

Cómo citar este artículo/How to cite this article: Gallardo-Frías, L. (2021). Propuestas para vincular la ciudad y sus habitantes con lugares de arte contemporáneo. Estoa. Revista de la Facultad de Arquitectura y Urbanismo de la Universidad de Cuenca, 10(20), 91101. doi: https://10.18537/est.v010.n020.a08 


\section{Referencias bibliográficas}

Almeida, P. X., Ayala, P., Cortez, K. y Tituaña, M. S. (2012) Arte y comunidad: Espacios de transformación. $A R Q$ (Santiago), (81), 62-66. https://dx.doi.org/10.4067/ S0717-69962012000200011

Álvarez Domínguez, P. y Benjumea Cobano, J. R. (2011). Aproximación al Museo Contemporáneo: Entre el templo y el supermercado cultural. Arte y Políticas de Identidad, 5, 27-42. https://revistas.um.es/reapi/article/ view/146201

Ayala, I. Cuenca-Amigo, M. y Cuenca, J. (2020). Examining the state of the art of audience development in museums and heritage organisations: a Systematic Literature review. Museum Management and Curatorship, 35(3),306-327. https://doi.org/10.1080/09647775.201 9.1698312

Badiou, A. (2013). Las condiciones del Arte Contemporáneo. [Conferencia] Universidad Nacional de San Martín.

Baldellou Plaza, Á. (2014). Museos: tesoros y ludotecas. $P+C$, (5), 33-46. https://repositorio.upct.es/bitstream/ handle $/ 10317 / 4366 / \mathrm{mtl}$.pdf?sequence=1\&isAllowed=y

Botte, J. Doyen, A. y Uzlyte, L. (2017). 'Ceci n'est pas un musée' : panorama géographique et historique des définitions du musée. En F. Mairesse, Définir le musée du XXle siècle. Matériaux pour une discussion. ICOFOM.

Bueno Carvajal, J. (2021). Espacio público e identidad Tres escenarios de interpretación en la ciudad contemporánea. Estoa. Revista de la Facultad de Arquitectura y Urbanismo de la Universidad de Cuenca, 10(19), 161 - 169. https://doi.org/10.18537/est.v010. n019.a14

Calaf Masachs, R. y Gutiérrez Berciano, S. (2016). E Museo Thyssen-Bornemisza: evaluando sus programas educativos para enseñar arte. Arte, Individuo y Sociedad, 29(1), 39-56. https://doi.org/10.5209/ARIS.49123

Cameron, D. (1971). The museum a temple or the forum Curator, the Museum Journal, 14 (2), 11-24. https://doi org/10.1111/j.2151-6952.1971.tb00416.x

Collados-Alcaide, A. (2014). Laboratorios artísticos colaborativos. Espacios transfronterizos de producción cultural. Arte, Individuo y Sociedad, 27(1), 45-64. https:// doi.org/10.5209/rev_ARIS.2015.v27.n1.43648

Corominas, J. (1987). Breve diccionario etimológico de la lengua castellana. Gredos.

Déotte, J-L. (2012). ¿Qué es un aparato estético? Benjamin, Lyotard, Rancière. Metales Pesados.

Desvallées, A. (2000). Pour une terminologie muséologique de base. La muséologie/museology (Cahiers d'étude/ Study series), 8. ICOM.
Dorrian M. (2014). Museum atmospheres: notes on aura, distance and affect. The Journal of Architecture, 19(2) 187-201. https://doi.org/10.1080/13602365.2014.913 257

Falcón Meraz, J.M. (2019). La arquitectura del museo: testigo y evidencia de la época. Arquiteturarevista, 8 (2) 135-147. https://doi.org/10.4013/arq.2012.82.04

Ferrer, J. Á. (2018). Rafael Moneo. El arte y la arquitectura de los museos. Diseño editorial.

Fiori Arantes, O. (2016). Gentrificación estratégica. REVISTARQUIS, 5(1), 103-121. https://revistas.ucr.ac.cr/ index.php/revistarquis/article/view/25406

Galland, D. (2014). Procesos y estilos de planificación en la rehabilitación urbana: el caso de Dinamarca. Revista de Arquitectura, 19(27), 15-24. https://doi. org/10.5354/0719-5427.2014.33564

Gallardo-Frías, L. (2014). Siete puntos de análisis en el proceso proyectual. El contexto urbano en el proyecto arquitectónico. Bitácora Urbano Territorial, 2(24). https://revistas.unal.edu.co/index.php/bitacora/article/ view/38667

Gallardo Frías, L., Toledo Jofré, M., Figueroa Garavagno, C., Vera Bravo, J. y Pérez Huenupi, L. (2019). Centro Nacional Arte Contemporáneo Cerrillos (CNACC): motor de transformación de la ciudad de Santiago. Revista de Urbanismo, (40). https://revistaurbanismo.uchile.cl/ index.php/RU/article/view/52362

Gallardo Frías, L. (2020). El lugar del arte. Reflexiones sobre conceptos para obras arquitectónicas destinadas al arte contemporáneo. Revista Anales del Instituto de Investigaciones Estéticas, 42(116), 9-27. https://doi. org/10.22201/iie.18703062e.2020.116.2713

García Fernández, I. M. (2015). El papel de los museos en la sociedad actual: discurso institucional o museo participativo. Complutum, 26(2), 39-47. https://doi. org/10.5209/rev_CMPL.2015.v26.n2.50415

Guasch, A. M. (2008). Los museos y lo museal: el paso de la modernidad a la era de lo global. Calle 14. Revista de investigación en el campo del arte, 2(2), 10-20. https://revistas.udistrital.edu.co/index.php/c14/article/ view/1236

Hernández Sánchez, A. y De La Torre Sánchez, C. (2021). Niñeces migrantes en el Centro Histórico de Puebla. Estrategias para la integración social. Estoa. Revista de la Facultad de Arquitectura y Urbanismo de la Universidad de Cuenca, 10(19), 77- 88. https://doi.org/10.18537/est. v010.n019.a07

Hernández Hernández, F. (1992). Evolución de concepto de museo. Revista General de Información y Documentación, 2(1), 85-97. https://revistas.ucm.es/ index.php/RGID/article/view/RGID9292120085A 
Huerta, R. (2018). Miradas urbanas del profesorado iberoamericano desde la cultura visual. Magis, Revista Internacional de Investigación en Educación, 10(21), 5576. doi: 10.11144/Javeriana.m10-21.mup

ICOM. Estatutos (2007). Artículo 3 - Definiciones, Apartado 1. Consejo Internacional de Museos. ICOM.

Kisida, B., Greene, J.P. y Bowen, D. H. (24 de noviembre de 2013). Arts makes you smart. The New York Times. https://www.nytimes.com/2013/11/24/opinion/sunday/ art-makes-you-smart.html

Layuno Rosas, M. Á. (2003). Museos de arte contemporáneo y ciudad. Los límites del objeto arquitectónico. En: J.P. Lorente y D. Almazán. (Ed.), Museología crítica y arte contemporáneo (pp. 109-123). Universidad de Zaragoza. Prensas Universitarias.

Luque Rodrigo, L. (2018). La ciudad contemporánea: problemas y tendencias. Perspectivas institucionales, sociales y artísticas. Sémata: Ciencias Sociais e Humanidades, 30, 377-398. https://doi. org/10.15304/s.30.5371

Mairesse, F. y Desvallées, A. (2007). Vers une redéfinition du musée ? L'Harmattan.

Marinetti, F. T. (20 de febrero de 1909). Le Futurisme. Le Figaro. Gómez de la Serna, R. (Trad.) (1909). Revista Prometeo, 2 (VI).

Newhouse, V. (1998). Towards a new museum. Monacelli Press.

Özkan Yazgan, E. y Akalin, A. (2019). Comprehension of place awareness in a historical context: metaphors in architectural design education. METU JFA. 36(1), 183202. http://jfa.arch.metu.edu.tr/archive/0258-5316/ articles/metujfa2019107.pdf

Poulot, D. (2009). Musée et muséologie. La Découverte.

Rispal, A. (2009). L'architecture et la muséographie comme médiation sensible. Muséologies, 3(2), 90-101. https://www.erudit.org/fr/revues/museo/2009-v3-n2museo02125/1033564ar.pdf

Rivière, G. H. (2009). La museología. Curso de museología textos y testimonios. Akal.

Rocco, F. (14 de agosto de 2018). Museums. Temples of delight. The economist. https://www.economist.com/ special-report/2018/08/14/temples-of-delight

Unesco (4 de abril de 2020). Los museos ante los desafíos de COVID-19 continúan comprometidos con las comunidades. Unesco. https://es.unesco.org/news/ museos-desafios-covid-19-continuan-comprometidoscomunidades

Valenzuela, F.A., Espinosa, A., Madero-Cabib, I., Moyano, C. y Ortiz, F. (2015). Los museos de arte como mecanismos de inclusión y exclusión social en el arte y en la sociedad: un estudio de caso en Chile. Boletim do Museo Paraense Emilio Goeldi. Ciencias Humanas. 10(3), 723-737.

Valéry, P. (2005). Piezas sobre arte. La balsa de la Medusa. Villarreal A. (2007). MUSBA: Museo de barrio. Revista 180. (19), 14-17. http://dx.doi.org/10.32995/rev180 Num-19.(2007).art-196

Weil, S. (1995). A cabinet of curiosities. Inquiries into museums and their prospects. Smithsonian Institution Press. 